\title{
Hendrikje Kilian:
}

\section{Der elektronische Lesesaal-Syka}

Im Februar dieses Jahres wurde mit dem Systematischen Katalog der letzte konventionelle Katalog im Hauptlesesaal abgebrochen. Seitdem bieten wir unseren Benutzern den Syka als elektronischen Katalog an. Damit konnte die zweijährige Entwicklungs- und Testphase erfolgreich zum Abschluss gebracht werden.

\section{Entwicklung des Katalogs}

Der elektronische Syka ist eine Eigenentwicklung unserer Bibliothek. Nachdem der Alphabetische Katalog des Hauptlesesaals als erster Sonderkatalog der WLB konvertiert worden war, sollte auch der Systematische Katalog in elektronischer Form abgebildet werden. Im August 1997 stellte die EDVAbteilung in einer mail "ihren Prototyp eines Systematischen Online-Katalogs für den Hauptlesesaal zur Ansicht und zur Diskussion". Die erste Testversion beinhaltete nur die Fachgebiete Technik und Astronomie, zeigte aber schon die drei Bestandteile des Katalogs, die über NETSCAPE untereinander verbunden sind:

- die Leitkartensystematik

- die Kurztitel der Bücher, die hinter einer Leitkarte beginnen (sortiert nach der LS-Notation)

- die Volltitel zu jedem Kurztitel

Die Leitkartensystematik wurde durch Eingabe in eine Textdatei abgebildet und dann automatisch in html-Seiten konvertiert. Für die Kurztitel wurden die Daten vom OLIX-OPAC abgezogen, formatiert und in eine Datenbank auf dem Linux-Server geladen. Dem Update-Rhythmus des OLIX-OPAC entsprechend werden diese Daten alle 14 Tage neu abgezogen und geladen.

$\mathrm{Zu}$ jedem Kurztitel kann der Volltitel aus unserem OLIX-OPAC abgerufen werden. Bisher benötigen wir hierzu noch den WWW-Server in Tübingen, der die Datenbank im RUS (Rechenzentrum der Universität Stuttgart) anzapft. Ab Sommer soll dies nicht mehr nötig sein.

Im Anschluss an diese erste Vorstellung des Katalog-Prototyps begann eine umfangreiche Testphase: mehrere Referenten gaben ihre Systematiken ein und begannen mit dem neuen Katalog zu arbeiten. Gleichzeitig testeten die MitarbeiterInnen des Hauptlesesaals den elektronischen Katalog auf seine Eignung als Dienst- und Revisionskatalog. Dass er diese Funktion übernehmen könnte, stand früh fest. In vielen Gesprächen formulierte das LSPersonal seine Anforderungen, die von der EDV-Abteilung durch Herrn Abele und Herrn Bouché schnell und zuverlässig umgesetzt wurden. Dank dieser guten Zusammenarbeit steht heute nicht nur ein übersichtlicher Benutzerkatalog, sondern auch ein sehr funktionaler Arbeits- und Revisionskatalog zur Verfügung.

\section{Suchfunktionen im Syka}

Die Startseite des Katalogs bietet dem Benutzer einen Überblick über die im Hauptlesesaal vertretenen Fachgebiete (Abb. 1). Von Aa Allgemeine Nachschlagewerke bis $Z$ Baden-Württem- 
Datei Bearbeiten Ansicht Gehe Communicator Hilfe

Zuruck $\quad$ Vor Neuladen Anfang Suchen Guide Drucken Sicherheit Stap

\section{Hauptlesesaal}

Stichwortsuche Signaturensuche Mitarheiterfunltionen

\section{Aufruf nach Systematik}

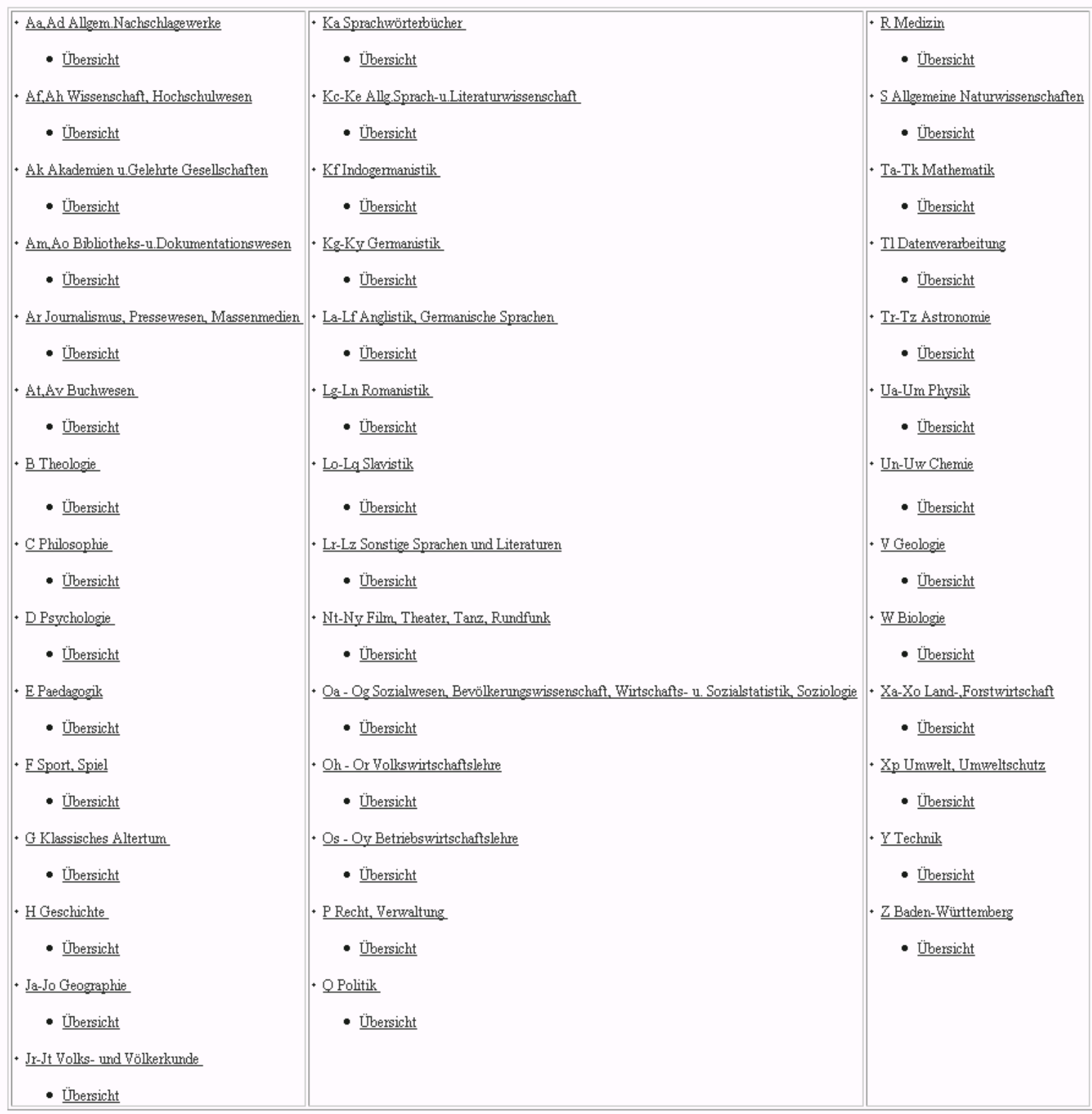


berg stehen 42 Fachgebiete zur Auswahl. Durch Anklicken des gewünschten Fachgebietes kann die Systematik in ihren einzelnen Hierarchiestufen aufgeblättert werden. Zusätzlich wird für jedes Fachgebiet noch eine Übersicht

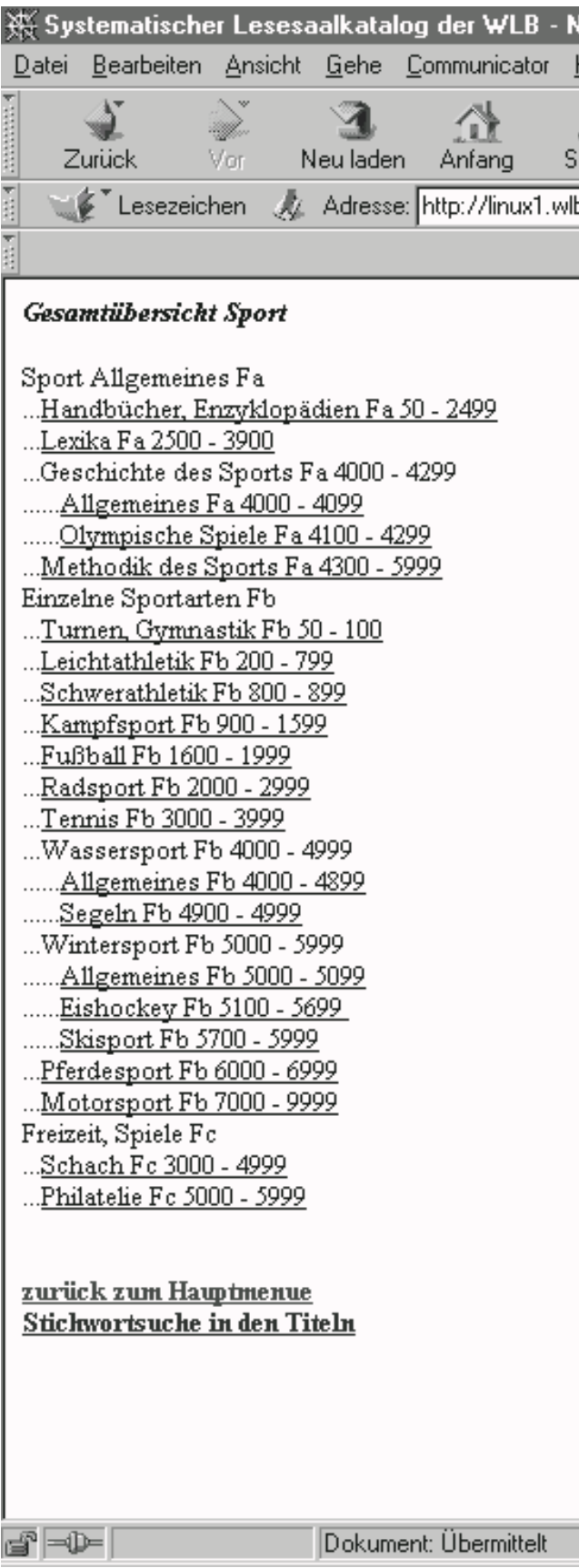

angeboten, die alle Hierarchiestufen auf einen Blick zeigt (Abb. 2). Auch von dort gelangt man durch Anklicken an die einzelnen Systemstellen.

An den jeweiligen Systemstellen werden die Titel in Listenform angezeigt (Abb. 3,).

Zusätzlich werden angegeben:

- LS-Signatur

- ggf. Hinweise ("am Schalter", beim Buchbinder u.ä.)

Durch Anklicken eines Titels erhält man die Volltitelanzeige (zu beachten: "Umgebung" des OPAC der UB Tübingen) (Abb. 4). Mit Hilfe des Buttons "Zurück" bzw. "Back" gelangt man in die jeweilige Hierarchiestufe der Systematik zurück.

Innerhalb einer Systemstelle findet man Angaben in verschiedenen Farben. Die Farben schwarz, rot, grün und braun kennzeichnen unterschiedliche Eintragungen:

- schwarz: Daten aus dem OLIXOPAC (d.h. bereits im LS befindliche Bücher)

- rot: Handeinträge Monographien (d.h. neue Bücher für den LS, die sich noch im Geschäftsgang befinden)

- grün: Verweisungen innerhalb des LS-Syka

- braun: Handeinträge Zeitschriften

- blau: 000-Datensätze (diese Eintragungen sind in der Benutzerversion nicht sichtbar)

Abbildung 2: Hierarchiestufen innerhalb eines Fachgebietes 
Innerhalb der Systematik bestehen folgende Navigationsmöglichkeiten:

- Vorwärtsblättern (um jeweils 1 Seite)

- Rückwärtsblättern (um jeweils 1 Seite)
- Gesamtliste (kein seitenweises Blättern, Systemstelle in Listenform angezeigt)

- eine Stufe zurück (auf die nächsthöhere Hierarchiestufe)

- zum Hauptmenü (zurück zur Startseite)

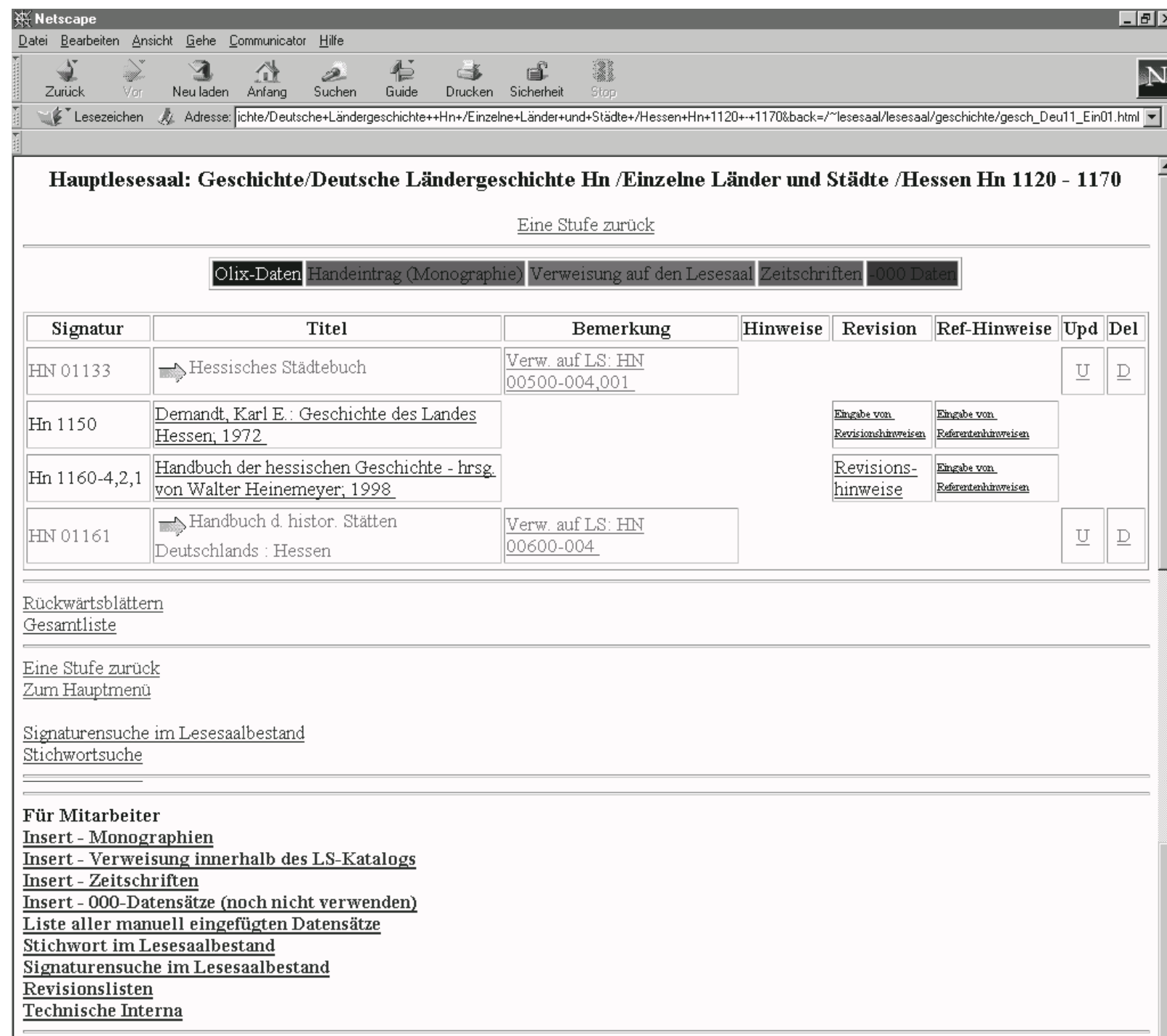


Neben der systematischen Suche werden noch die Stichwort- und die Signaturensuche angeboten (Abb. 5). Unter
Die Funktion Signaturensuche ermöglicht die Suche nach einer Lesesaalsignatur. Auch hierbei kann man mit Hilfe

Abbildung 4: Ergebnisanzeige

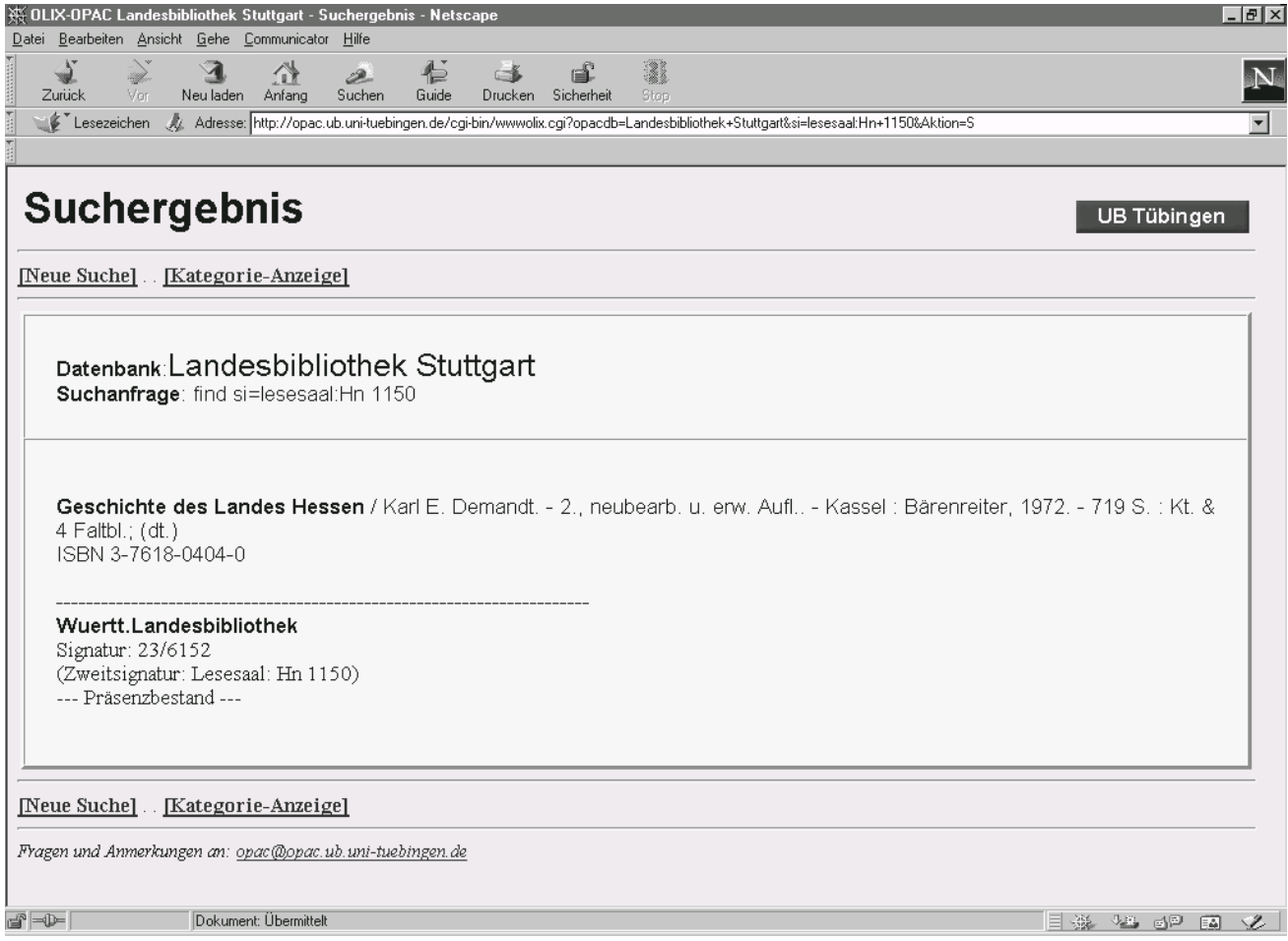

der Funktion Stichwortsuche kann nach Stichwörtern aus dem Titelfeld gesucht werden, wobei zu beachten ist, dass da im Titelfeld integriert - auch der Autor gesucht werden kann. Die Suchbegriffe können durch die logischen Operatoren UND bzw. ODER verknüpft werden. Trunkiert werden kann mit dem $\%$-Zeichen. Generell sind Links- und Rechtstrunkierungen möglich. Linkstrunkierungen sind allerdings sehr rechenintensiv und sollten möglichst vermieden werden. des \%-Zeichens trunkieren. Die Eingabe von Magazinsignaturen führt in diesem Katalog nicht zum Erfolg.

\section{Mitarbeiterfunktionen im Syka}

$\mathrm{Da}$ der elektronische Syka nicht nur den konventionellen Benutzerkatalog, sondern auch die konventionellen Dienstkataloge des Lesesaal-Personals und der Fachreferenten ersetzen soll, mussten zahlreiche Mitarbeiterfunktionen programmiert werden. Sie schaffen die Möglichkeit, Handeinträge vorzu-

Abbildung 5: Suchmaske für den LesesaalSyka

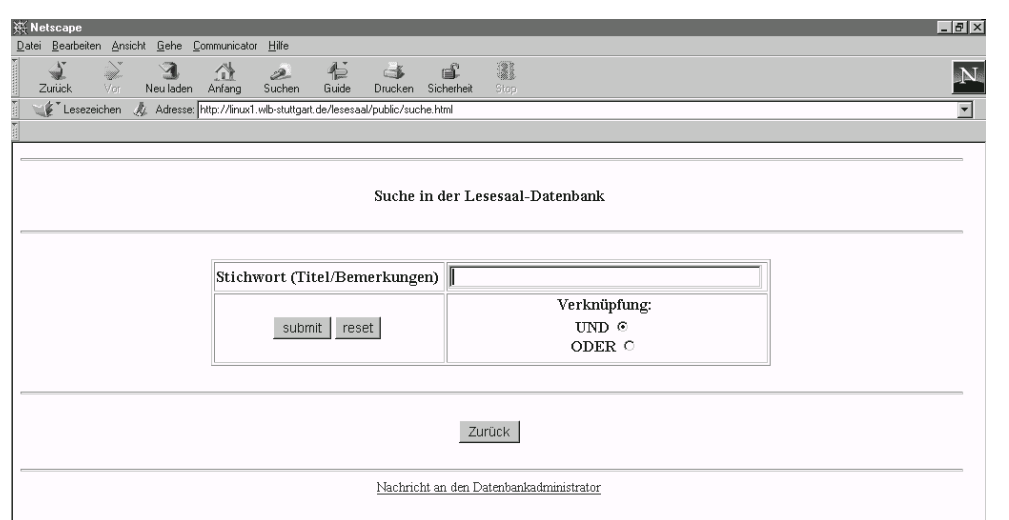


nehmen, eine Funktion, die sowohl für die Lesesaal-MitarbeiterInnen als auch für die Fachreferenten sehr wichtig ist.

Mitarbeiterfunktionen stehen nur an bestimmten Geräten, die von der EDVAbteilung explizit freigeschaltet werden müssen, zur Verfügung. Um mit diesen Funktionen arbeiten zu können, müssen Benutzername und Kennwort eingegeben werden.

Folgende Funktionen werden angeboten:

- Insert-Monographien

- Insert-Zeitschriften

- Insert - Verweisungen innerhalb des Lesesaalkatalogs

- Insert -000-Datensätze

- Liste aller manuell eingefügten Datensätze

- Revisionslisten

\section{Insert - Monographien}

Mit Hilfe dieser Funktion können die Fachreferenten Monographien, die in den Lesesaalbestand eingearbeitet werden sollen, in den Katalog eintragen. Das Eingabeformular sieht den Eintrag der LS-Signatur, des Kurztitels sowie ein Feld für Bemerkungen vor (Abb. 6).

Dieser Handeintrag erscheint zur besseren Unterscheidung in roter Schrift im Katalog (Abb. 7). Er ist auch in der Benutzerversion sichtbar, da die Information über neue Bücher auch für Benutzer interessant ist. Nach der Katalogisierung des Werkes wird der rote Handeintrag durch das Einspielen der Daten aus dem OLIX-OPAC überschrieben.

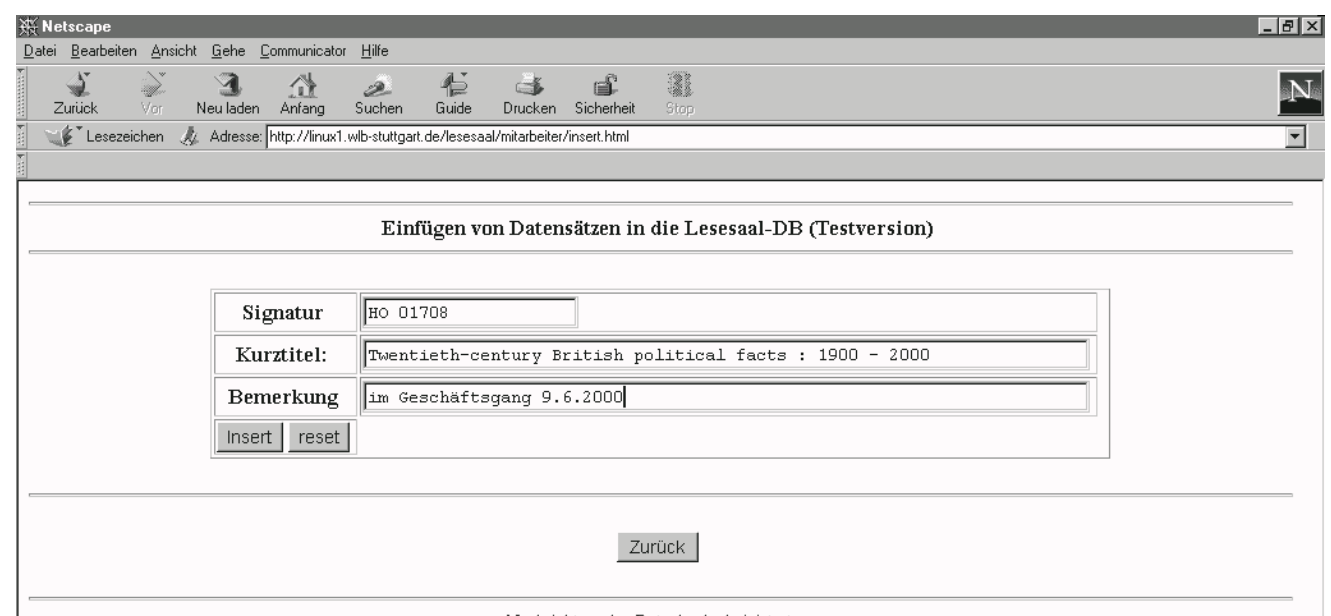

Nachricht an den Datenbankadministrator

Abbildung 6: Eingabeformular 


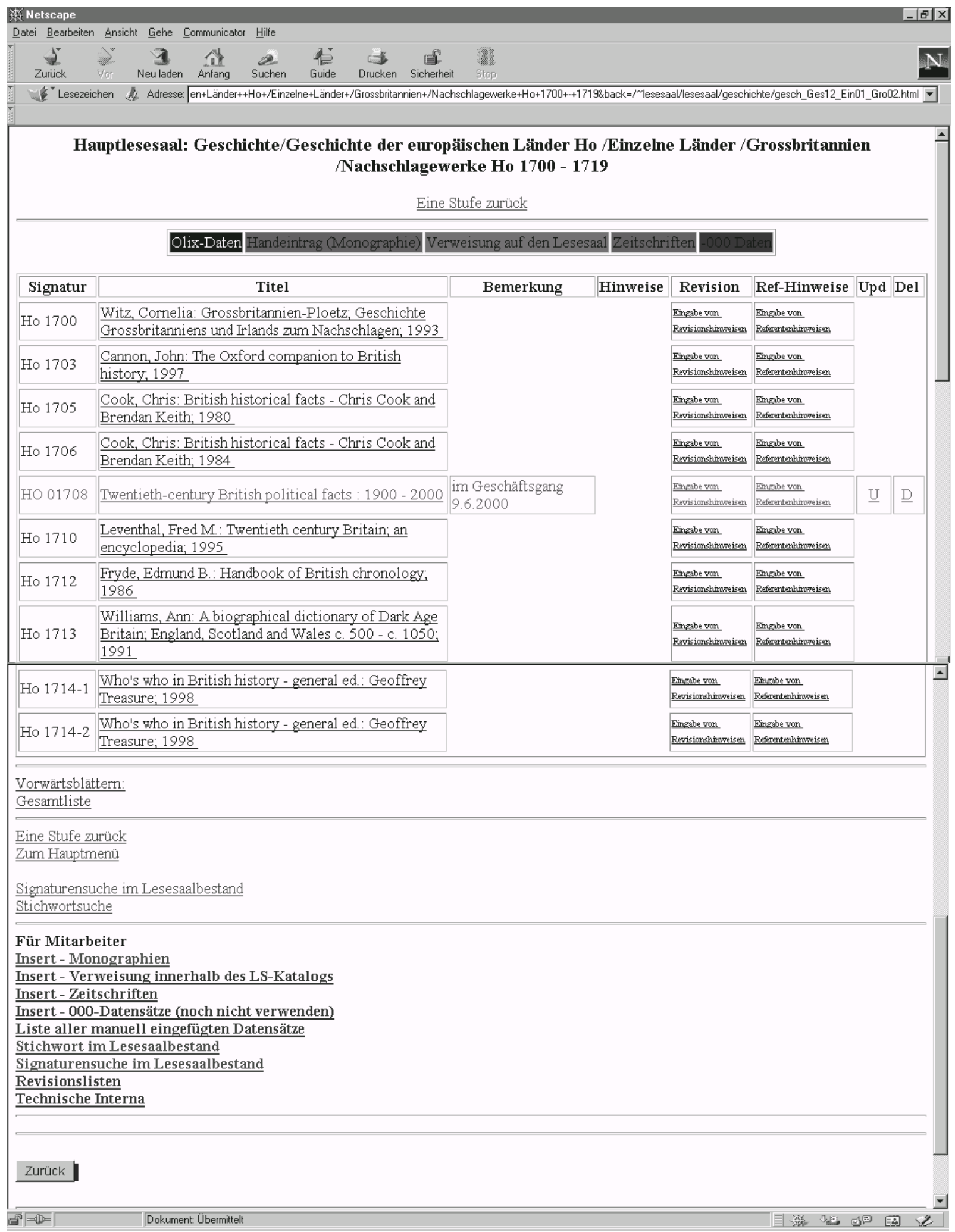

Abbildung 7: Handeintrag 


\section{Insert - Zeitschriften}

Diese Funktion ermöglicht das Einfügen der Zeitschriften durch Handeintrag in den Katalog. Da es derzeit noch nicht möglich ist, die Titelaufnahmen von Zeitschriften automatisch in den Lesesaalkatalog einzuspielen, mussten sie von Hand eingetragen werden. Für diesen Eintrag, der von den MitarbeiterInnen des Lesesaals vorgenommen wurde, wurde ein spezielles Eingabeformular programmiert. Eingetragen wurden die Lesesaal-Signatur, der Zeitschriftentitel sowie die im Lesesaal vorhandenen Bände. Der Handeintrag erscheint in brauner Schrift.

Da der Eintrag der Zeitschriften in einem eigenen Datenbankbereich erfolgt, ist eine leichte Selektion dieser Handeinträge möglich. Einer automatischen Einspielung der Zeitschriftentitel zu einem späteren Zeitpunkt steht daher nichts im Wege.

\section{Insert - Verweisungen innerhalb des Lesesaalkatalogs}

Verweisungen auf an einer anderen Systemstelle stehende Literatur kann der Fachreferent unter dieser Funktion eintragen. Damit die Verweisung an der inhaltlich richtigen Stelle im Katalog erscheint, muss auch für den Verweisungseintrag eine Signatur vergeben werden. Diese Pseudosignatur ist allerdings für den Benutzer nicht sichtbar. Die Benutzerversion zeigt nur den Kurztitel und die LS-Signatur des Werkes, worauf verwiesen wird.

Im Katalog erscheinen die Verweisungseinträge in grüner Schrift.

\section{Insert - 000-Datensätze}

Mehrbändige Werke werden im Katalog durch die Aufführung der einzelnen Bände dargestellt. In Einzelfällen fügen die LS-MitarbeiterInnen bei mehrbändigen Werken noch einen Handeintrag ein, der nur aus einer Pseudosignatur und einem Revisionshinweis besteht. Die Pseudosignaturen sind erkennbar an der daran angehängten 3fachen 000 (z.B. Hg 1480-000). Sie ermöglichen ein manuelles Eintragen der Bandzahl. Dies ist nötig in den Fällen, bei denen die automatische Zählung zu fehlerhaften Angaben in den Revisionslisten führen würde. Diese - für die Benutzer nicht sichtbaren - Handeinträge werden in blauer Schrift dargestellt.

\section{Liste aller manuell eingefügten Da- tensätze}

Unter dieser Funktion kann man sich eine vollständige Auflistung aller Handeinträge - selektierbar nach Monographien, Verweisungen, Zeitschriften und 000-Datensätzen - anzeigen lassen.

\section{Revisionslisten}

Diese passwortgeschützte Funktion steht nur den LS-MitarbeiterInnen zur Verfügung. Die Revisionslisten ersetzen die konventionelle Revisionskartei des Lesesaals. Man kann sich eine Gesamtliste, aber auch Listen von Teilbeständen (bis hin zu einzelnen Systemstellen) erstellen lassen.

Ohne diese Revisionslisten wäre die Durchführung der jährlichen Bestandsrevision nicht möglich. Sichtbares Ergebnis der Bestandsrevision ist die Lesesaal-Statistik, die Auskunft gibt über die Bestandszahlen am Beginn und am Ende eines Betriebsjahres sowie über die Veränderungen innerhalb der einzelnen Fachgebiete. 